\title{
Ingenieras civiles de la Universidad Autónoma de Aguascalientes, 1973-2018. ¿Mujeres a contracorriente?
}

\author{
Female civil engineers of the Autonomous University of \\ Aguascalientes, 1973-2018: Women against the current?
}

DOI: https://doi.org/10.32870/dse.v0i21.630

Marcela López Arellano*

\begin{abstract}
Resumen
La inserción de las mujeres en la educación superior en el mundo ha sido un proceso silencioso y lento a lo largo de casi dos siglos. Sin embargo, la entrada de mujeres en carreras consideradas "masculinas" como lo es la Ingeniería Civil, ha estado llena de adversidades, especialmente al cuestionar su decisión de ingresar a una profesión "para hombres", tales como las resistencias de sus familias y su círculo social, de sus profesores y compañeros varones en la universidad, y sus luchas por insertarse en los espacios laborales de la construcción. Diferentes estudios sobre mujeres e Ingeniería Civil en varias universidades de México han demostrado que el registro de mujeres en esta carrera sigue siendo bajo hasta hoy. Por lo tanto, a través de entrevistas con seis ingenieras civiles graduadas en diferentes generaciones de la Universidad Autónoma de Aguascalientes (1973-2018), el presente trabajo examina conceptos como la inclusión, la exclusión, las resistencias, y sus estrategias para la permanencia en la carrera que han elegido. Experiencias en las que ellas han estado inmersas como parte de modelos culturales en los que los discursos de género continúan definiendo los espacios apropiados para las mujeres y aquellos que son apropiados para los hombres.
\end{abstract}

Palabras clave: Mujeres - Ingeniería Civil - inclusión - exclusión - estrategias.

\begin{abstract}
The insertion of women in higher education in the world has been a silent and slow process that has taken place over almost two centuries. However, the entry of women into careers considered "masculine" such as Civil Engineering has been full of adversities, such as the resistances of their families and social circle, those of their male professors and male classmates at the university, as all of them question their decision to enter into a profession considered "for men", as well as their struggles to find spaces in the field of construction. Different studies about women and Civil Engineering in several universities in Mexico have shown that women's registration in this major continues to be low to this day. Therefore, through interviews with six women civil engineers graduated from different generations of the Autonomous University of Aguascalientes (1973-2018), this paper examines concepts
\end{abstract}

* Doctora en Ciencias Sociales y Humanidades/Historia. Profesora Investigadora en el Departamento de Historia, Universidad Autónoma de Aguascalientes. Líneas de investigación: Historia de mujeres y de género; Cultura escrita, Historia de Aguascalientes y México. México. maloarellano@hotmail.com, marcela.lopeza@edu.uaa.mx 
such as inclusion, exclusion, resistances and their strategies for permanence in the career they have chosen, experiences in which they have been immersed as part of cultural models in which gender discourse continues to define the appropriate spaces for women and those that are proper to men.

Key words: Women - Civil Engineering - inclusion - exclusion - strategies.

\section{Introducción ${ }^{1}$}

Los dos últimos siglos han sido testigo del lento proceso de inserción de las mujeres a la educación, especialmente a la educación superior universitaria. La investigadora Carmen Benso apunta que desde finales del siglo XIX las mujeres han protagonizado la "más importante revolución, social, silenciosa -y pacífica- de los últimos tiempos". El acceso a la educación superior ha representado para ellas el conocimiento, la reflexión, más libertad, autonomía y dignidad, ingreso en el mundo laboral y apertura a la emancipación femenina (Benso, 2003: 57-58). La historiadora Patricia Mazón refiere que la "admisión de mujeres a la educación superior no significó tan sólo eliminar las barreras para las mujeres, sino una selección cuidadosa de las mujeres que podrían ingresar en la universidad" (Mazón, 2003: 7).

Este proceso ha significado tensiones y rechazo de la sociedad hacia las mujeres, en muchos casos se consideró que invadían espacios y funciones netamente masculinas. El siglo XIX fue testigo de las propuestas y reglamentos educativos en los cuales la educación para los niños se planteó desde lineamientos científicos, y para las niñas, a partir de la necesidad de instruirlas como educadoras de sus hijos, los futuros ciudadanos. A lo largo de todo ese siglo se avanzó muy lentamente en la escolarización de las niñas, en la formación de las maestras y "en el derecho de las mujeres a realizar estudios superiores" (Subirats, 1994: 52). Durante el siglo XX las mujeres fueron abriendo camino en todas las carreras, tanto las femeninas como las consideradas masculinas. Actualmente, en pleno siglo XXI, en la mayoría de los países las mujeres pueden ingresar a la carrera que deseen y trabajar en el área que prefieran. No obstante, algunas carreras como la Ingeniería Civil, siguen siendo consideradas "sólo para hombres."

El presente trabajo tiene como objetivo examinar algunas de las resistencias familiares, culturales y sociales que han enfrentado las ingenieras civiles de la Universidad Autónoma de Aguascalientes (UAA) a lo largo de poco más de cuatro décadas (1973-2018), tanto como estudiantes y como profesionistas en el campo laboral. A través de la revisión de varios estudios sobre las ingenieras civiles en distintas universidades de México y de entrevistas a seis egresadas de la UAA, se muestran algunos elementos de la inclusión o exclusión a una profesión considerada como "masculina", desde las experiencias narradas por ellas y las estrategias que adoptaron para sostenerse en el campo de estudios y el trabajo de su elección.

1 Agradezco a la Dra. María Teresa Fernández Aceves (CIESAS-Occidente) su generosidad para revisar este texto y sus atentas recomendaciones. 


\section{Mujeres en las universidades en México}

En México, durante el siglo XIX el acceso de las mujeres a la educación superior y a las profesiones se desarrolló inicialmente en la capital mexicana, que era el centro intelectual del país, si bien con cuestionamientos acerca de la importancia de la educación de la mujer para formar y educar a su familia (Cano, 1996: 2). Las leyes de Instrucción de 1867 y de 1869 no marcaron impedimentos para que las mujeres ingresaran a la Escuela Nacional Preparatoria (ENP), requisito para acceder a las instituciones de educación superior, y desde 1888 algunas se inscribieron en la ENP, interesadas en estudiar Medicina, Derecho, Ingeniería, Telegrafía y el área de la salud (Alvarado, 2000: 12-14).

Además, cuando en 1881 el filósofo Justo Sierra ${ }^{2}$ presentó el proyecto para crear una universidad, precisó que las mujeres "tendrían el derecho a cursar todas las clases de las escuelas profesionales, obteniendo [...] diplomas especiales de la Escuela Normal y de Altos Estudios" (Alvarado, 2000: 13). Sin embargo, las restricciones respecto a los espacios permitidos para las mujeres las desanimaron de ingresar a las universidades, y no fue hasta el cambio de siglo y la Revolución mexicana que más mujeres se inscribieron en la Universidad Nacional, si bien la mayoría en Enfermería o en la Escuela Normal Superior (Galván, 1985: 36).

Como se ve, desde finales del siglo XIX las universidades abrieron poco a poco la matrícula para las mujeres y no solo a las carreras "femeninas" como las Ciencias Sociales y las Humanidades, sino también a Medicina, Derecho e Ingenierías — consideradas masculinas—, porque en la legislación universitaria en México no había estatutos que prohibieran el ingreso de las mujeres a ninguna de las carreras (Gutiérrez, 2015: 30). Vale mencionar que, en algunos países como España, las mujeres solo podían inscribirse en las universidades si los comités académicos lo autorizaban, en una condición previa de exclusión. Ellas estaban transgrediendo una norma no escrita, "el reparto de poder y saber entre los dos sexos" (Benso, 2003: 69-70), situación que, de acuerdo con Mazón, se dio también en Alemania, en donde para 1909 las mujeres podían ingresar a todas las universidades y lograr los mismos títulos que los hombres, pero la decisión de admitirlas dependía de los requisitos "discriminatorios" sustentados por los académicos varones (Mazón, 2003: 137).

En México, en 1910 se fundó la Escuela de Altos Estudios, con conocimientos científicos y filosóficos. En ese año las mujeres inscritas representaron 15\% del alumnado y para 1926, se incrementó en 78\%, lo cual es significativo en el acceso de las mujeres a la educación superior en México. Ellas se matricularon en magisterio, pero también en Biología, Botánica, Arqueología y Antropología. Fue en esta Escuela de Altos Estudios y en Filosofía y Letras que se desarrolló una "genealogía intelectual femenina [...] a lo largo de la primera mitad del siglo XX" (Cano,

2 Justo Sierra Méndez (1848-1912) Escritor, historiador, periodista, poeta, político y filósofo mexicano. Fue promotor de la fundación de la Universidad Nacional de México, hoy UNAM. 
1996: 178). Ya en la Universidad Nacional de México, los títulos para mujeres en Medicina y los de maestría, comenzaron a otorgarse en los años veinte y los de doctorado hasta la década de 1930. La primera egresada de Ingeniería Civil de esta institución fue Concepción Mendizábal Mendoza, quien ingresó en la Escuela Nacional de Ingeniería en 1926 y se tituló en 1930, con una tesis sobre una torre elevada de concreto armado (La Crítica, 2018).

En otro caso, en la Universidad de Guadalajara, que fue instaurada en 1925 por el gobernador Guadalupe Zuno, de acuerdo con la historiadora María Teresa Fernández, durante los primeros años las mujeres eligieron carreras "femeninas", como maestras y enfermeras, pero a finales de la década y durante la siguiente aumentaron su presencia en las carreras profesionales consideradas masculinas, muchas de las cuales, al egresar enfrentaron el rechazo de la sociedad y fueron llamadas "muchachas indecentes" (Fernández, 1995: 95-99). Tal vez por ello, la mayoría continuaron eligiendo profesiones femeninas, como dentista, enfermera, partera, farmacéutica y maestras, más como "una prolongación de las tareas doméstica [que] como una vocación" (Ibíd: 104). Lo que muestra que el debate sobre el ingreso de las mujeres a las universidades desde principios del siglo XX fue acerca de su inserción al campo de conocimiento científico masculino y sobre trabajar en los mismos espacios que ellos.

En la Universidad Autónoma de Puebla la historia parece repetirse, la historiadora Gloria Tirado la llama una "universidad altamente masculinizada" que en 1950 tenía alrededor de 2,500 alumnos y era "excepcional que [las mujeres] optaran por una carrera [...] masculinizada, como una ingeniería" (Tirado, 2009: 17-21). Para 1968, esta universidad ya tenía aproximadamente 11 mil alumnos y solo 17\% eran mujeres. La Facultad de Ingeniería Civil inició en 1869; en 1937 el Colegio se convirtió en Universidad de Puebla y la Facultad de Ingeniería fue conocida como"La isla de los hombres solos", a pesar de lo cual, la primera mujer egresó en 1974. (ibíd.: 37).

De igual forma, la investigadora Susana Gutiérrez, al analizar el ingreso de mujeres a la Escuela de Ingeniería Civil en la Universidad de Baja California (UBC), encontró que también fue conocida como "La isla de los hombres solos". Esta Escuela fue creada en 1967 en la ciudad de Mexicali y, a pesar del sobrenombre, desde la primera generación egresó una mujer en 1970 (Gutiérrez, 2015: vi). Llama la atención que tanto en Puebla como en Baja California conocieran a Ingeniería Civil como una isla solo para hombres. ${ }^{3}$

Gutiérrez observó que al ingresar a Ingeniería Civil las mujeres desarrollan estrategias y se apropian de los espacios. Encontró que las primeras estudiantes de Ingeniería Civil en la UBC llegaron con representaciones tradicionales sobre ser hombre y ser mujer, y se insertaron en una cultura estudiantil masculina en la que la ingeniería era exclusiva de hombres. La presencia de ellas, dice, contradijo este discurso de exclusión que "trastocaba las identidades, experiencia y subjetividad de las mujeres, en la búsqueda entre las representaciones sobre ser mujer y

3 Gutiérrez apunta que la referencia es a la novela de José León Sánchez, La isla de los hombres solos, publicada en 1963, sobre un penal para hombres en Costa Rica.

Diólo pos 
ser ingeniero." (Gutiérrez, 2015: vi-vii). La investigadora advirtió el énfasis en "la práctica" como elemento de la construcción de masculinidad de los ingenieros civiles en la UBC, y sobre el concepto de masculinadad se refiere a Connell, quien la define como "no es sólo una idea de alguien ni una identidad personal. Se extiende a lo largo de todo el mundo y se mezcla con relaciones sociales" (Connell, 2003: 51).

En 2015 las investigadoras Florentina Preciado, Karla Kral y María Guadalupe Álvarez, de la Universidad de Colima, publicaron un estudio acerca de las mujeres en el contexto de la ingeniería en aquella institución, entre las cuales estuvieron las ingenieras civiles. Destacan que para 2011 la distribución nacional de la matrícula por sexo y área, según el Anuario Estadístico de ANUIES, mostró que las mujeres en ingenierías y tecnologías eran $29 \%$. Sin embargo, sostienen que, si bien las mujeres han aumentado en las áreas masculinizadas como las ingenierías, estos estudios "siguen siendo considerados como 'un tema de hombres', ya que la socialización e identidades de género, tanto en la familia como en la escuela, refuerzan la noción de que los hombres 'naturalmente' tienen habilidades para 'temas difíciles' como las matemáticas y la ciencia" (ibíd.: 43-44). Y manifiestan que las estudiantes mexicanas se enfrentan a diferentes discursos "sobre el hecho de ser mujer en relación con la ingeniería" (ibíd.: 45).

A esto se suma el estudio de la investigadora Patricia Angélica García Guevara, de la Universidad de Guadalajara, en el cual constató que, de acuerdo con el Departamento de Control Escolar de la UDG, "para el ciclo 2015-2016 a la carrera de Ingeniería Civil ingresaron 85.15\% de hombres y $14.85 \%$ de mujeres" (García Guevara, 2019: 3).

\section{Mujeres en Ingeniería Civil en la Universidad Autónoma de Aguascalientes}

La Universidad Autónoma de Aguascalientes fue fundada el 19 de junio de 1973 (UAA, 2015). ${ }^{4}$ En sus primeros diez años ofreció 22 licenciaturas, una técnica superior y dos carreras técnicas. La carrera de Ingeniería Civil se abrió en 1974 y en 1975 tenía una mujer inscrita en el tercer semestre. Este fue un año interesante porque en la capital mexicana tuvo lugar la I Conferencia Mundial del año Internacional de la Mujer en México, que tuvo la educación de las mujeres como objetivo (ONU-Mujeres). Sin embargo, no fue hasta 1984, a diez años de su fundación, cuando se tituló la primera mujer de Ingeniería Civil.

La investigadora Claudia Castellanos examinó el ingreso de dos generaciones de mujeres a la UAA y no localizó reglamentos o requisitos que limitaran su acceso. Para contextualizar su estudio apunta que, en 1971, en la Universidad Nacional Autónoma de México, 20\% de las mujeres se inscribieron en la Facultad de Filosofía y Letras, 17\% en Medicina, 13\% en Comercio, 12\% en Derecho, 2\% en Arquitectura y solo $0.4 \%$ en Ingeniería (Castellanos, 2015: 16-20). Lo que parece mostrar que la ingeniería no era una de las carreras preferidas por las estudiantes.

4 La UAA tiene su origen en la Escuela de Agricultura que inició en 1867, luego fue el Instituto de Ciencias del Estado; en 1942 se convirtió en el Instituto Autónomo de Ciencias hasta 1973, cuando fue fundada como universidad. 
Al revisar el crecimiento de la población estudiantil en la carrera de Ingeniería Civil en la UAA, Castellanos encontró que en 1975 ingresaron 31 alumnos y en 2012 había 381, en un crecimiento sostenido desde su inicio. No obstante, observó que la población femenina en esta carrera ha mantenido niveles bajos a lo largo de más de cuatro décadas, de un inicial $3 \%$ en la década de 1970 a 7\% en 1985, llegando a ser 12\% en 2012 (Ibíd.: 110-112). ${ }^{5}$ En otras carreras de esta institución que iniciaron con un bajo ingreso de mujeres, como Veterinaria, el registro de mujeres subió de $3 \%$ en 1975 a 40\% en 2012, y Agronomía, que inició con 12\% en sus primeros años, aumentó a 43\% en 2011 (Ibíd.: 109-114). La investigadora señala que al analizar los porcentajes de alumnos y alumnas en las distintas carreras de la universidad, observó que "la población femenina [aumentó] dependiendo del área de conocimiento [pero que] siguen existiendo carreras que históricamente han sido y siguen ocupadas por hombres, como Ingeniería Civil" (Ibíd:: 22).

Actualmente el ingreso de mujeres en Ingeniería Civil en la UAA presenta porcentajes similares. En entrevista con un profesor de la carrera, este señaló que en julio de 2017, de un grupo de 36 estudiantes se graduaron cinco ingenieras, y en diciembre de ese año, de un grupo de 29, egresaron otras cinco. Refirió que, en julio de 2018, de un grupo de 29 estudiantes, salieron dos ingenieras; en la generación que se graduó en diciembre de 2018, que contó con 33 estudiantes, egresaron dos mujeres; y en la que concluye sus estudios en julio de 2019, de 50 estudiantes, se tiene un "histórico" 6 de diez mujeres. Finalmente, en el grupo que egresó en diciembre de 2019, que son 17 alumnos, sólo tres son mujeres.

El académico destacó que la carrera de Ingeniería Civil logró la Certificación ABET (Accreditation Board for Engineering and Technology) en 2012, y aunque "se observó un aumento en la demanda de estudiantes, no significó un mayor número de mujeres" (Profesor Ingeniero Civil, 2018). Como se ve, según estos datos, en seis generaciones solo han egresado o llegado a los últimos semestres 28 mujeres que, en un aproximado de 205 estudiantes, representan 13\% en años recientes, muy similar a los datos examinados por Castellanos hasta el año 2012.

\section{Inclusión-exclusión, resistencias, modelos culturales/estrategias}

A partir de esto surgen cuestionamientos acerca de los factores que inciden en la baja inscripción de mujeres a Ingeniería Civil. Entre estos, se pueden considerar la "inclusión o la exclusión" desde la perspectiva institucional y cultural, como lo expone la historiadora Carmen Benso, y las "resistencias" $y$ "modelos culturales" que agrupan el conjunto de prejuicios que existen en la sociedad, como lo formula la socióloga Marina Subirats que apunta, "las mujeres acceden cada vez más a la educación formal, pero ello no supone que tengan las mismas posibilidades que los hombres." (Subirats, 2018: 201). Esto es significativo porque a lo largo de varias décadas han

5 Claudia Castellanos tuvo acceso a los datos del Departamento de Estadística de la UAA.

6 Así lo calificó el entrevistado.

Diólo@os sobre Educación año 11 | número 21 | julio-diciembre 2020 | ISSN 2007-2171 
tenido lugar cambios sociales y culturales en México y en el mundo, sobre todo con la apertura de opciones de estudio y campos laborales "para hombres" en los que ellas han decidido incursionar.

De acuerdo con Benso, históricamente la pretensión de las mujeres de ingresar a las universidades implicó tres problemáticas, primero la apertura de las instituciones, luego la validez académica de sus estudios, y finalmente, "la posibilidad de ejercer la profesión para la que facultaba el título académico" (Benso, 2003: 12-13). En el caso de la UAA se aprecia que desde su inicio ha permitido la inscripción de mujeres a todas las carreras e igualmente otorga validez a su título, por ello vale examinar el binomio inclusión-exclusión desde las siguientes preguntas: ¿cuáles son los principales factores que han obstaculizado la inscripción de mujeres en la carrera de Ingeniería Civil en la UAA?, ¿han sido estos de orden normativo o cultural?, ¿cuáles han sido las estrategias de las estudiantes para mantenerse en la carrera?, ¿de qué manera los modelos culturales han afectado su inserción en el campo laboral?

Al problematizar el concepto de inclusión, con su contraparte de exclusión, se advierte que, dentro del problema de la inequidad en el acceso de las mujeres a la educación superior, la variable de acceso o inscripción por sí sola no muestra la inclusión o exclusión a los espacios del conocimiento. Se presenta una aproximación a las experiencias de las ingenieras civiles, ya no solo desde su ingreso a la carrera, sino desde distintos tipos de exclusión y de resistencia, que van de lo familiar, social y cultural a la esfera de los poderes en el espacio público.

\section{Ingenieras civiles, trayectorias}

Se realizaron seis entrevistas temáticas a ingenieras civiles egresadas de la Universidad Autónoma de Aguascalientes en las generaciones de 1984, 1989, 1993, 1995, 2014 y 2016. ${ }^{7}$ Se seleccionaron por haber realizado sus estudios en diferentes generaciones $y$, especialmente por estar dedicadas hasta la actualidad al campo laboral de la ingeniería civil, ya sea en la obra civil, en oficinas gubernamentales dedicadas a la obra pública y en constructoras de allegados o familiares. Las edades de las entrevistadas van, de alrededor de los 50 años para las egresadas de las primeras generaciones hasta los 26 para las más recientes.

Si bien en las entrevistas surgieron temas como su situación familiar, si son solteras, casadas o divorciadas y también la maternidad, para este trabajo las preguntas se enfocaron a su decisión para estudiar esta carrera, el contexto universitario y su inserción en el campo laboral. Sus testimonios permiten conocer el contexto familiar en la elección de carrera y la influencia de la escolaridad de sus padres en ello, las experiencias al interior de la institución con sus compañeros y profesores varones, y la búsqueda de oportunidades de trabajo en la ingeniería civil. Sus narrativas muestran la reproducción del discurso de género, que permite apreciar que esta profesión sigue siendo considerada como un espacio "de hombres."

7 Para este artículo no identifico a las ingenieras entrevistadas con su nombre real, las cito con un seudónimo y la generación en la que egresaron. 
Vale destacar que las seis entrevistadas de la UAA se designan como ingenieras, ${ }^{8}$ contrario a lo encontrado por la investigadora Susana Gutiérrez en entrevistas con egresadas de ingeniería civil de la Universidad Autónoma de Baja California, para quienes apropiarse del título de "ingeniero" les significó la igualdad entre hombres y mujeres dentro del campo (Gutiérrez Portillo y Duarte Godoy, 2012). Sobre la forma de nombrarse a sí mismas, la investigadora Susana Guerrero ha señalado que "la Real Academia recomienda feminizar y masculinizar los términos que aludan a oficios y profesiones [que los] títulos o certificados deben adecuarse al sexo de las personas que los obtengan [sea] ingeniero/ingeniera" (Guerrero, 2014: 28-29). Como se ve, algunas ingenieras lo han comenzado a hacer a pesar de que en sus papeles académicos quedaron como "ingenieros".

A partir de las seis entrevistas se identificaron siete apartados que muestran elementos de la inclusión, la exclusión y las resistencias familiares, culturales y sociales hacia el campo de estudio y de trabajo que ellas eligieron. Como contraparte, también se hicieron visibles las estrategias con las cuales ellas se mantuvieron en el espacio universitario elegido, y han permanecido en el campo de trabajo que les apasiona. Estos apartados son: la elección de la carrera, el ingreso a la institución universitaria, el lenguaje como práctica cultural, la imagen, el espacio, la inclusión y participación, y el campo laboral.

\section{La elección de carrera / Ingeniería Civil}

La historiadora Gloria Tirado registra que "casi siempre" quienes estudian las carreras de ingenierías, física y matemáticas, han sido alentadas por "figuras masculinas que ayudan a que rompan estereotipos, roles tradicionales y sobre todo se familiaricen con las ciencias exactas" (Tirado, 2009: 35). Sin embargo, en las presentes entrevistas una de ellas refirió que tuvo que rebelarse a su padre que se opuso a la Ingeniería Civil por ser "para hombres" (Adriana, 1995), no obstante, las otras cinco entrevistadas tuvieron un padre o un abuelo con conocimientos en la construcción o las matemáticas. Para Ana (1989), su padre extranjero la impulsó a estudiar lo que ella quisiera, como lo hacían las mujeres en Europa; para Azucena (1993) la inspiración fue su abuelo que se dedicaba a la construcción; Aitana (2014) tomó como modelo a su padre arquitecto, y Amaya (2016), enamorada de los puentes y carreteras desde niña, recibió todo el apoyo de su padre maestro.

En este apartado vale señalar que una de las mayores resistencias que enfrentaron las ingenieras al elegir su carrera fue de la sus madres y abuelas, en muchos casos les argumentaron que "era un espacio para hombres, no para señoritas" (Aranza, 1984; Ana, 1989; Adriana, 1995). Ninguna de las entrevistadas tenía otras ingenieras en la familia, y su decisión implicó romper las tradiciones familiares acerca del estudio "propio" para mujeres, lo cual abunda en los discur-

8 En el diccionario de la Real Academia Española de la Lengua se indica que es masculino y femenino: ingeniero e ingeniera. Ellas señalan que son ingenieras, aunque sus títulos universitarios dicen Ingeniero Civil.

Diálo pos sobre Educación año 11 | número 21 | julio-diciembre 2020 | ISSN 2007-2171 
sos sociales repetidos una y otra vez por los amigos y las familias, que "constriñen las acciones de las estudiantes dentro del campo" (Gutiérrez Portillo y Duarte Godoy, 2012: 4).

Es interesante observar cómo lo enseñado desde la familia acerca de lo considerado femenino y lo masculino prevalece. Frases como "es carrera de hombres", "¿en qué vas a poder trabajar después?", "es para hombres ¿quién te va a querer dar trabajo?" (Ibíd.: 7), convierten la decisión de las jóvenes en una lucha entre lo aprendido en casa y su vocación por los materiales, las matemáticas y la construcción —como lo definieron varias-, y que además las obligó a evaluar sus deseos contra los discursos tradicionales encarnados en las mujeres que fueron sus modelos de feminidad al crecer.

Por ejemplo, Aranza (1984) refirió que durante toda la carrera su madre la presionó para que se saliera y buscara otra carrera más adecuada a mujeres; Ana (1989) señaló que su abuela materna, a la que nunca le gustó que estudiara ingeniería civil, le repitió continuamente que "cómo una mujer va a mandar a los hombres", que si "quería convertirse en albañil", y Adriana (1995), cuyo padre se opuso a su elección, señala que su madre no la apoyó, se quedó callada secundando la oposición del esposo. No obstante, para Azucena (1993) cuya madre estudió Nutrición en la UNAM, y para Amaya (2016) cuya madre era maestra, la elección de carrera no supuso contraponerse a sus familias. Lo cual también permite reflexionar acerca del impacto que la escolaridad de las mujeres en la familia puede tener en la libertad para la selección de estudios por parte de las hijas y nietas.

En su investigación sobre las ingenieras civiles en la Universidad Autónoma de Baja California, Susana Gutiérrez apunta que el ethos de la Ingeniería Civil está ligado a "máquinas pesadas, fuerza física e independencia del hogar", con prácticas en la obra, viajes y campamentos como "representaciones del ingeniero que utiliza la fuerza física [opuesto] al rol tradicional de la mujer y a las características de la feminidad" (Gutiérrez, 2015: 43). Es interesante observar que las resistencias que experimenta la mayoría de las mujeres al elegir esta profesión reflejan las concepciones de género, sociales y culturales de las familias y el entorno social. Vale destacar que las seis entrevistadas, a pesar de pertenecer a distintas generaciones, recurrieron a la estrategia de mantenerse firmes ante las opiniones adversas a sus deseos e ingresaron a Ingeniería Civil en la UAA.

\section{El ingreso}

Como ya se mencionó, en la UAA no existe una normatividad explícita que les impida a las mujeres el acceso a las carreras que elijan, por tanto, todas tuvieron el campo abierto para inscribirse y presentar los exámenes correspondientes para ser aceptadas. En algunos casos recordaron que se les pedía haber cursado el área de físico-matemáticas en los bachilleratos, aunque no era requisito obligatorio. Sobre esto, la investigadora Patricia Angélica García Guevara señala "que la participación de las mujeres en carreras que anteriormente eran consideradas 'masculi- 
nas' o 'exclusivas' para los hombres está aumentando [aunque] la Ingeniería Civil, la Mecánica e Ingeniería en Comunicación y Electrónica, son las que históricamente tienen menor presencia femenina" (García Guevara, 2019). Esta autora encontró que en 1999 la participación femenina en Ciencias Exactas e Ingenierías en las universidades de México fue de 23\%, y que la población escolar de la carrera de Ingeniería Civil en la Universidad de Guadalajara en 2000-01, tuvo 92\% de hombres y $8 \%$ de mujeres (García Guevara, 2002: 92-95).

Al revisar los índices de ingreso de mujeres a Ingeniería Civil en varias universidades, se aprecia que los porcentajes son similares y vale preguntarse: ¿por qué esta carrera no es elegida por más mujeres? ¿por qué otras carreras anteriormente consideradas "masculinas" como Ingeniería en Agronomía o Medicina, Veterinaria y Zootecnia han crecido en población femenina y la ingeniería civil no?

Sobre el bajo porcentaje de ingreso de mujeres a esta carrera, las seis entrevistadas de la UAA respondieron desde sus propias visiones: Aranza (1984) dijo "creo que actualmente ya no es tanto por el machismo, pero es una carrera pesada y las matemáticas no son fáciles, además de que tiene que gustarles mucho la construcción"; Ana (1989) piensa que "las jóvenes tienen miedo del trato con los hombres y les dan miedo las matemáticas, además que desde niñas las educan para que estudien cosas de niñas"; Azucena (1993) señaló que cree que no entran más chicas porque "no saben que la ingeniería abre muchas puertas, cubrimos muchos perfiles, en las matemáticas, como profesoras, como constructoras, y más"; Adriana (1995) recuerda que en la UAA decían que "había tres clases de mujeres, las feas, las bonitas y las de Ingeniería Civil", piensa que eso desanima a muchas y que las niñas mexicanas no quieren ser ingenieras; Aitana (2014) cree que hace falta ir a las escuelas a contarles a las niñas que ser ingenieras "es emocionante e implica muchos retos que ellas pueden realizar, incluidas las matemáticas"; y Amaya (2016) respondió que se debe a que "no ha cambiado la idea de que el espacio de trabajo es difícil para ellas".

Son interesantes las concepciones de ingenieras con experiencia en el campo laboral de la construcción, dejan ver los modelos culturales en los cuales se sigue diferenciando lo "adecuado" para ellas, en contraste con lo que es "propio de hombres", y parece no haber una gran diferencia entre lo que manifestaron las egresadas en la década de los años ochenta y lo expresado por las que se graduaron en la segunda década del siglo XXI.

Vale añadir que para este trabajo se revisaron los programas de estudio de las distintas carreras que se encuentran en la página web de la UAA, y se advierte que las comunicaciones se dirigen a el egresado, el estudiante, el aspirante. Modelos también de lenguaje y de pensamiento que siguen permeando en la diferenciación de lo que es para unos y para otras.

\section{El lenguaje como práctica cultural}

Según lo refieren las ingenieras, la exclusión se denota en la interacción cotidiana con algunos maestros y sus compañeros varones que las descalifican con los comentarios. En su estudio, 
Castellanos observó que las relaciones interpersonales entre alumnas y maestros, y alumnas con sus compañeros varones en la UAA, mostraron "cierto rechazo por parte de los profesores a las alumnas en carreras como [...] Ingeniería Civil: [...] "¿qué hacían allí? [...] debían estar en su casa lavando platos o cocinando" (Castellanos, 2016: 31). Por otro lado, la investigadora Guadalupe Contreras, en su tesis sobre feminización de la matrícula en la UAA entrevistó a otra ingeniera egresada en 1984, quien refirió la resistencia de sus padres para estudiar una carrera "para hombres", así como las resistencias de algunos profesores en la carrera, uno de ellos les dijo: "qué grupo tan populoso de mujeres [iniciaron seis, y dos se salieron en el primer semestre] les va a ir muy mal... todas reprueban... no pasan del primer semestre" (Contreras, 2015: 136).

Las ingenieras entrevistadas, para este capítulo igualmente expresan que experimentaron resistencias de los compañeros varones y de algunos de sus profesores por ser mujeres, desde el inicio y durante la carrera, en los salones, en sus prácticas de campo y en el servicio social o prácticas profesionales en el área de la construcción (Aranza, 1984; Ana, 1989; Azucena, 1993; Adriana, 1995; Aitana, 2014; Amaya, 2016). Aranza (1984) recordó que en el segundo semestre de la carrera eran cuatro mujeres y a su grupo "lo conocían como el grupo de las muchachas porque éramos muchas mujeres."

De acuerdo con Tirado, la masculinización se nota en las prácticas culturales como el lenguaje. En su investigación sobre las estudiantes de ingeniería en Puebla, ellas le dijeron haber escuchado repetidamente frases como:"es Ud. prófuga del metate", "muestre las manos para ver si sabe cocinar", "a lo que Ud. viene es a buscar marido", "Ud. estudia mientras se casa” (Tirado, 2009: 71). Expresiones similares a las que las ingenieras de la UAA recibieron de algunos profesores: "Ud. no va a poder, las matemáticas son para hombres" (Aranza, 1984); “Las mujeres son para ir a su casa a atender a su marido y ponerse a lavar" (Ana, 1989); "¿Qué hace Ud. aquí? Esto es para hombres, las mujeres se hicieron para lavar y planchar en su casa" (Adriana, 1995); "Ud. debería estar en la cocina haciendo caldito de pollo" (Aitana, 2014); "Ud. no pasa del quinto semestre, esto es para hombres" o"si no puede con esto, mejor ponga una tiendita de uñas" (Amaya, 2016). Una forma de exclusión desde los ámbitos académicos en donde algunos profesores, ellos mismos ingenieros, a partir de sus propias concepciones de la ingeniería como un espacio masculino, descalifican la decisión de ellas de pertenecer a este campo de trabajo.

No obstante, es importante destacar que las entrevistadas también refirieron la ayuda que recibieron de otros profesores ingenieros, las apoyaron en sus estudios, les dieron ánimos, las recomendaron para algún trabajo, o las emplearon en sus empresas constructoras. Las seis recordaron experiencias como, "gracias al ingeniero [...] que expresó su confianza en mí, me puse a estudiar más y logré graduarme" (Azucena, 1993); "el ingeniero [...] confiaba en mí, me decía, ve y diles que te enseñen a manejar la motoconformadora" (Amaya, 2016).

Esta dicotomía permite reflexionar acerca de las cambiantes concepciones sociales y culturales alrededor de lo que se considera masculino y lo que se identifica como femenino, y cómo 
es posible observarlas dentro de la educación superior, en donde el lenguaje como forma simbólica expresa la asimetría de las relaciones de género y la reafirmación de las masculinidades, o la apertura desde la perspectiva contraria. En cualquiera de los casos, vale reflexionar que los discursos cargados de doble lenguaje como: "la mujer debe ser sumisa, abnegada, femenina," las presionan para demostrar todo el tiempo que ellas son capaces de aprender los "saberes masculinos", distintos a los femeninos (Tirado, 2009: 81).

\section{La imagen: botas y chaleco}

Otro aspecto interesante de observar es la necesidad que la mayoría de las entrevistadas de la UAA tuvieron de "vestirse como ellos" (Aranza, 1984; Azucena, 1993; Adriana, 1995; Aitana, 2014; Amaya, 2016) como una estrategia frente a las resistencias de sus compañeros. Manifestaron que ponerse botas de construcción, ropa floja y chalecos se convirtió en su escudo contra burlas, chiflidos, piropos o comentarios despectivos como "con esa falda quieres que el profe te ponga diez" (Amaya, 2016). A pesar de la distancia de cuatro décadas entre sus generaciones, es significativo que vestirse así les permitió pertenecer, sentirse "colegas", no mujeres. Tirado apunta que la forma de vestir es otro símbolo de la comprensión de la realidad, una estrategia para mezclarse con sus compañeros, borrar las diferencias entre lo masculino y lo femenino (Tirado, 2009: 81). Por tanto, vestirse "femeninas" las excluía. Azucena (1993) señala "conozco muchas ingenieras que literalmente andan vestidas como hombres", y a Adriana (1995) le dijeron "es que eres muy modosita, vas a tener que andar vestida para construir".

Sobre sus experiencias con los compañeros varones, las entrevistadas recuerdan cómo lograron "demostrar" sus aptitudes matemáticas y su "fortaleza" en los trabajos en campo. Una refiere que le cuestionaban sus calificaciones, "para ellos yo era una mujer tonta" (Adriana, 1995). Narran las formas en que entretejieron redes de trabajo y amistad con algunos de ellos, en donde el alcohol y el lenguaje también significaron inclusión, "algunas se ponían a tomar igual que ellos para que las aceptaran" (Azucena, 1993). En sus recuentos se aprecia que la representación del ingeniero con botas, chaleco y gorra, el lenguaje rudo y el alcohol, fueron modelos que siguieron algunas de las estudiantes para pertenecer al grupo de "ingenieros". Estrategias de inclusión que les permitieron cruzar los porosos límites sociales y culturales que definen las diferencias entre lo adecuado para ellas y lo que no.

\section{El espacio ¿otra isla de los hombres solos?}

Como punto significativo, varias ingenieras se expresaron sobre el lugar en que esta institución (la UAA) ubicó los salones para Ingeniería Civil desde principios de la década de 1990. Cuentan, "las aulas estaban hasta el fondo" (Azucena, 1993); "estábamos allá en la orilla donde casi sólo había hombres" (Adriana, 1995), o "cuando caminaban por allí chicas de otras carreras, salían a chiflarles" (Amaya, 2016). El espacio marginal como una ubicación simbólica masculina de las concepciones de género. Gutiérrez enfatiza la importancia del lugar en donde son ubicados los salones de clase para ciertas

Diólo@os

sobre Educación

TEMAS A año 11 | número 21 | julio-diciembre 2020 | ISSN 2007-2171 
carreras como una "construcción simbólica y los cambios y rupturas en las representaciones" (Gutiérrez, 2015:5). En este caso, la percepción de las entrevistadas fue la inquietud de estar transgrediendo límites territoriales definidos en función de ellos.

\section{Inclusión y participación}

Otro elemento de inclusión es la posibilidad de participación de las estudiantes en los comités de estudiantes o jefaturas de grupo. Sobre esto, Tirado apunta que encontró que generalmente las mujeres no aparecen en los consejos directivos, su organización es horizontal y de colaboración con los líderes. Señala que en los años sesenta, las estudiantes de Ingeniería Civil de la Universidad de Puebla debieron demostrar su capacidad y ganarse los espacios (Tirado, 2009: 75-79).

Sin embargo, en las entrevistas a las ingenieras de la UAA, la mayoría decidieron hacerse cargo de la jefatura de grupo y organizaron los viajes o la graduación. En varios casos señalan que sus compañeros las tomaron como "secretarias" para hacer estos trabajos (Aranza, 1984; Azucena 1993; Adriana 1995; Amaya, 2016). Una de ellas organizó la fiesta de graduación y sus compañeros varones le decían "lo que quieras poner, no importa, eres mujer, tú sabes hacer eso" (Amaya, 2016). En este apartado surgen varias reflexiones acerca de los modelos culturales a través del tiempo. Las entrevistadas estudiaron en contextos históricos distintos, podría suponerse que, si para las estudiantes de los años setenta y ochenta ingresar a la carrera supuso luchar a contracorriente con la sociedad, para las estudiantes del siglo XXI los espacios abiertos por las primeras serían distintos. Sin embargo, al revisar sus relatos se advierte que la lucha por ganar reconocimiento y respeto en esta profesión parece la misma para todas, y vale preguntarse lo siguiente: ¿Cómo ven actualmente los estudiantes varones a sus compañeras de carrera? ¿las concepciones de género, en las cuales se afirma que Ingeniería Civil es para hombres, siguen transmitiéndose de generación en generación? ¿este patrón de exclusión familiar, social y cultural se repite en los salones de clase de las universidades en pleno siglo XXI?

\section{Enfrentarse al campo laboral / Inclusión en espacios públicos}

Las entrevistas revelan los diversos retos que enfrentaron las seis ingenieras en sus decisiones ante un campo de trabajo considerado masculino. Apuntan que ser ingenieras tituladas de una de las instituciones más reconocidas en Aguascalientes, como es la UAA, no les otorgó de inicio un respeto de los colegas en su campo laboral y menos de los trabajadores de la obra. En la mayoría de los casos debieron utilizar un lenguaje "masculino", con palabras fuertes y posturas beligerantes ante hombres que les cuestionaron que fueran las "jefas", las "supervisoras" o las "residentes" en la obra. Adriana (1995) apunta, "llego a la obra y me olvido que soy mujer, soy un constructor más, para ponerte enfrente de un grupo de albañiles y de un grupo de empresarios, debes olvidarte de que eres mujer." 
A través de sus narraciones se advierte que para todas las ingenieras la relación con los albañiles ha resultado complicada, "algunos se ponen agresivos y hasta nos sacan el cuchillo" (Aranza, 1984). Una recuerda, "la primera vez que llegué a una obra todos los albañiles me empezaron a chiflar... me convertí en ellos con su vocabulario y no me volvieron a faltar el respeto" (Ana, 1989); a otra le espetaron: ¿cómo? ¿una vieja que venga y me mande?" (Azucena, 1993). Todas escucharon una y otra vez frases como: "a mí no me va a mangonear una mujer", "usted no es el ingeniero", "las viejas no saben nada de ladrillos".

Su incursión laboral con colegas ingenieros tampoco ha sido sencilla, la mayoría entró a trabajar a los mismos lugares que sus compañeros de clase, pero a ellos los colocaron como supervisores y a ellas como ayudantes con un sueldo menor. Una afirma, "una mujer tiene que dar más, demostrar que es más responsable y que puede hacer todo el trabajo" (Aranza, 1984). Otra señala: "creo que soy la supervisora que más obras ha supervisado... he sobresalido con mucho esfuerzo y trabajo... y no he llorado bajo presión, porque pierdo toda la fuerza que he ganado ante ellos" (Ana, 1989). Una ingeniera recuerda, "un jefe me humilló ante los albañiles, les dijo que era mujer y no sabía" (Aitana, 2014), otra expresó, "el director de supervisión de una obra en la que yo era residente, me acosaba y decidí renunciar" (Adriana, 1995), y otra señaló "un auxiliar de laboratorio de materiales no me recibió el trabajo porque yo era mujer" (Amaya, 2016).

En sus experiencias se advierte que han requerido templanza y perseverancia para continuar en el área laboral que eligieron, sus estrategias han sido no cejar y mantenerse firmes a toda costa. Una constante interesante en sus relatos es el valor que este sentido de responsabilidad ha dado al trabajo de las mujeres, como apuntó una, "me piden que les recomiende ingenieras porque han visto que trabajan mejor" (Amaya, 2016).

En Aguascalientes existe El Colegio de Ingenieros Civiles, A.C. y la Cámara de la Industria de la Construcción (CMIC), espacios públicos organizados de los profesionistas relacionados con la construcción. Sin embargo, ninguna ingeniera civil ha tenido acceso a los puestos de dirección. Una señala "en el momento que quise ser presidenta, no tuve apoyo" (Azucena, 1993). Otra recuerda "quisimos abrir un espacio para mujeres en el Colegio de Ingenieros para aportar a la sociedad siendo mujeres, y nos dijeron:'allí están las esposas de los ingenieros, pueden sentarse con ellas"' (Aranza, 1984).

Además, aunque las seis ingenieras civiles entrevistadas trabajan en el área de la construcción, sienten que la presencia femenina en esta área es muy escasa en Aguascalientes. No conocen mujeres ingenieras que hayan destacado a nivel local, regional o nacional. Tampoco cuentan con un grupo, asociación u organización en donde ellas puedan conocer sus trayectorias y trabajos, así como unirse para proponer proyectos que consideren pertinentes para la sociedad.

Para finalizar este apartado, resulta interesante mencionar que, en mayo de 2018, en Aguascalientes fue publicado el número 76 de la Revista Construye. Arquitectura. Ingeniería Civil. Urbanis- 
mo, dedicada al tema "Mujeres construyendo equidad". En el interior se encuentra un artículo que dice "Es indispensable una mayor participación de las mujeres en la CMIC", y otro artículo titulado "Mujeres construyendo equidad". Éste último presenta a cuatro mujeres que laboran en la construcción, una es Maestra en Diseño Urbano, la otra es administradora, la tercera es mercadóloga y la cuarta es Licenciada (Revista Construye, 2018: 16, 19-25). Resulta paradójico, después de todo lo visto en este trabajo, que no hayan incluido a las ingenieras civiles.

\section{A modo de conclusión}

En el artículo "Las mujeres y las ingenierías", los investigadores Oliveros Ruiz, Cabrera, Valdéz y Schorr, de la Universidad Autónoma de Baja California, señalan que en la actualidad las mujeres son 25\% respecto a los hombres en ingenierías, y apuntan que según ANUIES, para 2015 las mujeres fueron $30 \%$ en las áreas de Ciencias, Tecnología, Ingenierías y Matemáticas (STEM) (Oliveros et al., 2016: 90-92). También informan que el Primer Encuentro Nacional de Jóvenes en la Ingeniería, realizado en México en 2015, se trató el tema de las mujeres la en ingeniería, la discriminación, los tabúes sobre su incapacidad en las matemáticas y, sobre todo, la ausencia de modelos de mujeres científicas e ingenieras que motiven a las niñas a contemplar la posibilidad de estudiar ingeniería (Ibíd.: 92) Esto es interesante porque son los temas que las ingenieras trataron en las entrevistas, pero como se vio, la inscripción femenina en Ingeniería Civil en la UAA no ha aumentado más allá de 12 o 13\% a lo largo de cuatro décadas, aún no puede verse como un espacio de educación superior feminizado.

En la revisión de distintos trabajos sobre el tema se advierte que la Ingeniería Civil sigue siendo una carrera considerada de y para hombres, mayormente por su concepción del uso de la fuerza y el trabajo en espacios externos. El campo laboral requiere de ellas liderazgo ante los trabajadores de la construcción, muchos de ellos pertenecientes a clases sociales en las cuales el "machismo" - mencionado por todas las ingenieras - , se refleja en una notoria defensa de las concepciones de género que consideran que la mujer debe permanecer en su casa y no convertirse en la "jefa" de ellos.

Es una elección de educación superior altamente demandante, las aspirantes a ingenieras civiles deben demostrar su vocación y seguridad ante sus familias; una vez inscritas en la carrera, deben demostrar a los profesores que las cuestionan y a sus compañeros varones en clases que ellas son capaces de cumplir con la carga curricular al igual que todos. Y ya egresadas, deben demostrar en el mundo del trabajo que ellas tienen la capacidad, la voluntad y los conocimientos para pertenecer y ser incluidas en los ámbitos de la construcción, ya sea en la obra civil o en los grupos de profesionistas del ramo.

A cuatro décadas de las primeras egresadas de Ingeniería Civil de la UAA parece que permanecen invisibles, situación que se presenta como una experiencia compartida por las ingenieras civiles egresadas de otras universidades en México. Es interesante señalar que través de las entrevistas para el presente capítulo las seis ingenieras cayeron en cuenta de muchas de las situaciones 
que habían enfrentado, que en su momento sortearon sin reflexionar si era válido que se les cuestionara su elección, y ahora quisieran que las siguientes generaciones de jóvenes ingenieras no tuvieran que luchar por lo mismo.

Han propuesto la creación de estrategias de apoyo para estudiantes y egresadas de la carrera desde dentro de los espacios académicos, y a través de redes de comunicación en las cuales puedan servir de soporte entre ellas. Sugieren que la propia institución podría crear talleres de inclusión y participación para alumnas y alumnos de Ingeniería Civil en los cuales se conscientizara que la elección de la profesión es libre y personal, sin restricciones por ser un espacio para hombres. Vale revisar la propuesta de la Dra. Dolores Marisa Martínez Moscoso titulada ¿Ingeniera?, ¿enfermero? Diálogo con estudiantes para vivir la equidad de género en la universidad, que editó el Centro de Estudios de Género de la Universidad de Guadalajara en 2014, con talleres para docentes y escolares con el objetivo de invitar a los estudiantes a comprender las prácticas culturales en las que vivimos y la forma como es posible relacionarse con todas las personas sin establecer diferencias entre ellas (Martínez Moscoso, 2014).

Finalmente, queda su preocupación de apoyar proyectos en común y hacer visibles las aportaciones de las ingenieras en el país para que la sociedad les reconozca sus esfuerzos y trabajo, especialmente, proponen diseñar estrategias para acercar a las niñas a modelos de mujeres en la ciencia, que conozcan las distintas propuestas de educación superior a los que ellas pueden acceder y donde podrán contribuir al desarrollo de sus propias comunidades.

\section{Entrevistas:}

Profesor Ingeniero Civil UAA / 4 abril 2018.

\section{Ingenieras Civiles:}

Ana / 29 abril 2018 / Generación 1989.

Aranza / 30 abril 2018 / Generación 1984.

Amaya / 1 mayo 2018 / Generación 2016.

Azucena / 2 mayo 2018 / Generación 1993.

Adriana / 7 mayo 2018 / Generación 1995.

Aitana /9 mayo 2018 / Generación 2014.

\section{Referencias}

Alvarado, M. de L. (2000) Abriendo brecha. Las pioneras de las carreras liberales en México. Revista de la Universidad de México, (596), 11-17.

Benso Calvo, C. (2003). Exclusión, discriminación y resistencias: El acceso de la mujer al sistema educativo (1833-1930). En Jato Iglesias, E.; L. Iglesias da Cunha (eds.). Xénero e educación social. Santiago de Compostela: Edición Laiovento.

Diólopos 
Cano Ortega, R. G. (1996). De la Escuela Nacional de Altos Estudios a la Facultad de Filosofía y Letras, 1910-1929. Un proceso de feminización (Tesis doctoral). México: UNAM.

Castellanos Méndez, C. (2016). Género y educación superior. Trayectorias escolares de mujeres de dos generaciones de la Universidad Autónoma de Aguascalientes (Tesis doctoral). Aguascalientes: UAA.

Connell, R. (2003). Masculinidades. México: UNAM.

Contreras Cervantes, M. G. (2014). Mujeres y educación superior: Feminización de matrícula, elección de carrera y trayectoria universitaria en la Universidad Autónoma de Aguascalientes (Tesis de maestría). Aguascalientes: UAA.

Fernández Aceves, M. T. (1995). Las mujeres graduadas en la Universidad de Guadalajara, 19251933. En C. Castañeda (ed.). Historia social de la Universidad de Guadalajara. México: UdG/ CIESAS, 90-106.

Galván Lafarga, L. E. (1985). La educación superior de la mujer en México, 1876-1940. México: Cuadernos de la Casa Chata/SEP.

García Guevara, P. A. (2002). Las carreras en Ingeniería en el marco de la globalización: una perspectiva de género. Revista Latinoamericana de Estudios Educativos, 32(3), 91-105. México: Centro de Estudios Educativos.

García Guevara, P. A. (2015). Más participación de las mujeres en las ingenierías. Nota de Universidad de Guadalajara. Guadalajara: Centro Universitario de Ciencias Exactas e Ingenierías. http://www.udg.mx/es/noticia/mas-participacion-de-mujeres-en-las-ingenierias

Guerrero Salazar, S. (2014). Guía orientativa para el uso del lenguaje y de la imagen en la Universidad de Málaga. https://www.uma.es/media/tinyimages/file/GUIA ORIENTATIVA USO LENGUAJE Y DE LA IMAGEN UNIVERSIDAD DE MALAGA.pdf

Gutiérrez Portillo, S. (2015). "Nunca fue la isla de los hombres solos”: Representaciones de género y las primeras egresadas de la Escuela de Ingeniería en la Universidad Autónoma de Baja California (1967-1990) (Tesis doctoral). México: CIESAS-Occidente.

Gutiérrez Portillo, S.; M. M. Duarte Godoy (2011). Ingenieras o ingenieros: cómo se conciben las mujeres en el campo de ingeniería. Ponencia presentada en IX Congreso Iberoamericano de Ciencias, Tecnología y Género, en Sevilla, España, 31 de enero al 3 de febrero de 2012.

Martínez Moscoso, D. M. (2014). ¿Ingeniera?, ¿enfermero? Diálogo con estudiantes para vivir la equidad de género en la universidad. Guadalajara: Universidad de Guadalajara.

Mazón, P. (2003). Gender and the Modern Research University: The Admission of Women to German Higher Education 1865-1914. Stanford: Stanford University Press.

Mujeres construyendo equidad (2018). Revista Construye. Arquitectura. Ingeniería Civil. Urbanismo, (76), 16-25.

Oliveros Ruiz, M. A., E. Cabrera Córdoba, B. Valdés Salas y M. Schorr Wiener (2016). La motivación de las mujeres por las carreras de Ingeniería y Tecnología. Entreciencias 4(9), 89-96. 
ONU (s/f). Conferencias mundiales sobre la mujer. ONU Mujeres. http://www.unwomen.org/es/ how-we-work/intergovernmental-support/world-conferences-on-women

Soto Espinosa, A. J. (2018). Seis pioneras de la ingeniería en México. La Crítica. https://www.lacritica.org/ciencia/6-pioneras-de-la-ingenieria-en-mexico/

Subirats, M. y C. Brullet (1988). Panorámica sobre la situación educativa de las mujeres: análisis y políticas. En M. Subirats y C. Brullet (eds.), Rosa y azul: la transmisión de los géneros en la escuela mixta. España: Ministerio de Cultura, Instituto de la mujer. http://www.ub.edu/SIMS/ pdf/PensarDiferencias/PensarDiferencias-14.pdf

Subirats, M. (1994). Conquistar la igualdad: la coeducación hoy. Revista Iberoamericana de Educación, 6, 49-78.

Subirats, M. (2018). Cómo educar en igualdad de género. Entrevista El País. España. https:// aprendemosjuntos.elpais.com/especial/como-educar-en-igualdad-marina-subirats/

Tirado Villegas, G. (2009). Abriendo brecha. Mujeres universitarias poblanas del siglo XX. México: BUAP.

UAA (2015). Síntesis histórica de la Universidad Autónoma de Aguascalientes. http://www.uaa.mx/ nu/histog.php 